\title{
Advances in Ketogenic Diet Therapies in Pediatric Epilepsy
}

\author{
Battamir Enkhtuy, MD ${ }^{1 *}$, Hye Eun Kwon, MD ${ }^{2,}$, Heung Dong Kim, MD \\ ${ }^{1}$ Department of Neurology, Mongolian National University of Medical Sciences, Ulaanbaatar, Mongolia \\ ${ }^{2}$ Department of Pediatrics, International St. Mary's Hospital, Catholic Kwandong University College of Medicine, Incheon, Korea \\ ${ }^{3}$ Division of Pediatric Neurology, Department of Pediatrics, Severance Children's Hospital, Yonsei University College of Medicine, Seoul, Korea
}

Received: September 30, 2019

Revised: November 15, 2019

Accepted: December 2, 2019

Corresponding author:

Heung Dong Kim, MD

Division of Pediatric Neurology,

Department of Pediatrics, Severance

Children's Hospital, Yonsei

University College of Medicine, 50

Yonsei-ro, Seodaemun-gu, Seoul

03722, Korea

Tel: +82-2-2228-2060

Fax: +82-2-392-9118

E-mail: hdkimmd@yuhs.ac

* These authors contributed equally to the manuscript as first author.
Ketogenic diets are high in fat, low in carbohydrates, and contain an adequate amount of protein. In addition to the classic ketogenic diet, three alternative types of ketogenic diet therapies (KDTs) have emerged. In addition to clarifying the indications for early treatment using KDTs, ongoing research over the past decades has led to the recognition of their contraindications and adverse effects. Recent studies focusing on the targeted therapeutic range of KDTs are expected to elucidate the precise mechanisms by which they alleviate certain epilepsy syndromes and other disorders. In this review, we discuss recent advances in KDTs, focusing on six issues: the selection of a specific KDT; the use of KDTs for febrile infection-related epilepsy syndrome and super-refractory status epilepticus; the use of KDTs for infants with refractory epilepsy; links between the gut-brain axis and KDTs; triheptanoin; and the use of KDTs for disorders other than pediatric epilepsy.

Keywords: Diet, ketogenic; Drug resistant epilepsy; Child

\section{Introduction}

Ketogenic diet therapies (KDTs) are the first-line treatment for glucose transporter 1 deficiency syndrome (Glut1DS) and pyruvate dehydrogenase deficiency. KDTs are also an early course of treatment for several epilepsy syndromes, including Dravet syndrome, West syndrome (WS), and epilepsy with myoclonic atonic seizures, and should be offered to children who fail to benefit from two anti-epileptic drugs (AEDs) [1]. Based on clinical experience and research over the last decade, Kossoff et al. [1] updated recommendations for the use of dietary therapies for pediatric epilepsy in 2018 to include new indications, including febrile infection-related epilepsy syndrome (FIRES)/super-refractory status epilepticus (SRSE), Angelman syndrome, complex 1 mitochondrial disorders, and Ohtahara syndrome. The updated recommendations also state that most centers prefer a non-fasting state at initiation of a KDT, making hospital admission optional; that although a diet can be selected from four major KDTs, the classic ketogenic diet (KD) is associated with a higher likelihood of a seizure-free outcome for children under 2 years of age, whereas alternatives to the classic $\mathrm{KD}$ are favored for adolescents and adults; and that additional lab tests (e.g., selenium, free and total carnitine) and electroencephalography (EEG) should be performed.

High-fat/low-carbohydrate diets induce production of ketone bodies (KBs), which become the primary source of energy for cell metabolism instead of glucose [2]. KDTs have a broad-spectrum therapeutic range than medications due to their multi-mechanism properties, with KBs directly inhibiting vesicular glutamate trans-

Copyright(C) 2019 Korean Child Neurology Society

This is an Open Access article distributed under the terms of the Creative Commons Attribution Non-Commercial License (http://creativecommons.org/licenses/by-nc/4.0/) which permits unrestricted non-commercial use, distribution, and reproduction in any medium, provided the original work is properly cited. 
port, altering metabolism by inhibiting glycolysis and increasing mitochondrial adenosine triphosphate (ATP) production, activating ATP-sensitive potassium channels to prevent neuronal excitability, and increasing polyunsaturated fatty acid and decreasing reactive oxygen species by stimulating mitochondrial uncoupling proteins $[3,4]$. Thus, KDTs not only inhibit neuronal hyperexcitability but also have neuroprotective effects that correct cellular energy failure and guard against epileptic brain damage [3]. Some researchers anticipate that KDTs will be replaced by drugs that mimic the actions of KDTs or directly stimulate ketogenesis in the liver, making these dietary treatments more similar to pharmacological therapy [5-7]. At present, however, this possibility seems questionable because different KDTs involve different subsets of anti-seizure mechanisms that can be targeted to individual patients. In this review, we focus on recent advances in KDTs for pediatric epilepsy considering the following issues: selection of a specific KDT; use of KDTs for status epilepticus; use of KDTs for infants with refractory epilepsy (RE); links between the gut-brain axis and KDTs; triheptanoin; and use of KDTs for disorders other than pediatric epilepsy.

\section{Selection of a Specific KDT}

As compliance with the strict regimen of the classic $\mathrm{KD}$ is difficult, more flexible alternative variants have been employed. In addition to the classic $\mathrm{KD}$, three other major dietary treatments- the modified Atkins diet (MAD), low glycemic index treatment (LGIT), and medium chain triglyceride (MCT) diet—are now available for patients with epilepsy.

The MAD typically consists of a 1:1 to $1.5: 1$ ketogenic ratio, achieves more than $50 \%$ seizure reduction in two-thirds of children with RE [8-11], and is well tolerated by adolescents and adults [12] and those who do not adhere to the classic KD. Some studies report that the MAD is as effective as the classic $\mathrm{KD}$; although, the classic $\mathrm{KD}$ is associated with a higher likelihood of seizure freedom in children under 2 years of age with RE $[13,14]$ and epileptic individuals with myoclonic atonic seizures [13]. In addition to its advantages in regard to growth and physical abilities, the MAD can be a good option for long-term maintenance for Glut1DS patients [15]. Nevertheless, for infants with Glut1DS, the classic $\mathrm{KD}$ is still considered superior in the early course of treatment and is recommended for long-term maintenance if possible [1].

The LGIT involves swapping high glycemic index (GI) foods for low GI alternatives. The GI describes the tendency of foods to increase blood glucose, compared with an equivalent amount of reference carbohydrate, usually glucose. Thus, the LGIT uses a liberalized but still low carbohydrate intake, with carbohydrates sup- plied only in the form of low GI foods and allows a more flexible lifestyle for patients by permitting increased intake of carbohydrates [16]. It achieves around 50\% seizure reduction in half of pediatric patients with $\operatorname{RE}[17,18]$ and is useful for those who cannot tolerate the classic $\mathrm{KD}$ or $\mathrm{MAD}$ [17]. Considering its high efficacy, the LGIT is used as an alternative or supplementary treatment for Angelman syndrome $[19,20]$.

The MCT diet, which produces MCT (C6-12) that are more ketogenic than long-chain triglycerides, was introduced by Huttenlocher in 1971 [21]. The efficacy of the MCT diet is comparable to that of the classic $\mathrm{KD}$; over half of children achieve more than $50 \%$ seizure reduction with good tolerance and few side effects $[22,23]$. In animal models with MCT diet, $\mathrm{KB}$ concentrations in blood plasma are poorly correlated with seizure control [24,25], and there is a lack of evidence that KBs participate in stopping seizures [26]. This suggests that MCTs, rather than KBs, block seizure onset and raise the seizure threshold [27-29]. MCTs, which consist of approximately $60 \%$ octanoic acid (C8) and 40\% decanoic acid (C10), exert anti-seizure effects via $\alpha$-amino-3-hydroxy-5-methyl-4-isoxazolepropionic acid receptor (AMPA) receptor inhibition [26] as well as peroxisome proliferator-activated receptor $\gamma(\operatorname{PPAR} \gamma)$ activation and mitochondrial biosynthesis [29] by using decanoic acid rather than octanoic acid [26,29,30]. In an animal model of acute seizures, decanoic acid increased seizure thresholds in both the $6 \mathrm{~Hz}$ stimulation test (a model of drug-resistant seizures) and maximal electroshock test (a model of tonic-clonic seizures), although it did not block pentetrazol-induced seizures (proposed to be a model of absence seizures) [30]. Decanoic acid is considered to be a PPAR $\gamma$ agonist that increases brain mitochondrial function and ATP synthesis, thereby increasing seizure threshold $[29,31]$, but it does not alter glycolytic enzymes [32]. MCTs have the direct and selective action of inhibiting AMPA receptors in an animal model, which has been considered to be the first targeted anti-seizure mechanism of the MCT diet [26]. This gives rise to the question of whether the MCT diet can be replaced by AMPA receptor-blocking agents such as perampanel [33]. However, a recent study showed a synergistic interaction between perampanel and decanoic acid, as perampanel binds at a different AMPA receptor site than decanoic acid [34].

\section{Use of KDTs for Status Epilepticus}

Although FIRES and SRSE are included in the updated recommendations, the efficacy of KDTs was first shown for refractory status epilepticus (RSE) as described by Kossoff and Nabbout [35] in 2013. At that time, 10 retrospective studies including 32 children and adults with RSE showed dramatic beneficial effects of 
KDTs, with $78 \%$ of patients becoming seizure-free and most responding within 7 to 10 days. Patients with FIRES are also reported to be good responders to KDTs [36].

Recent studies with 10 or more patients report good outcomes of KDTs regardless of etiology and a low rate of complications in critically ill patients with RSE/SRSE [37-40]. For instance, more than half of patients achieved more than 50\% seizure reduction within a median of 7 days, desired ketosis was reached within a median of 4 days, and most patients successfully weaned off continuous infusion of anesthesia within 2 weeks after initiation of a KDT. Relatively low rates of adverse effects were noted, but these included gastrointestinal disturbances, electrolyte imbalance, and ketoacidosis, with the main causes of discontinuation being pancreatitis and hypertriglyceridemia $[38,39]$. These outcomes are consistent with those of other studies with fewer than 10 patients [41-49], and patients with FIRES have also been found to respond quickly after KDT initiation [40,46,47]. Most patients are good responders, weaning off infusion and successfully reaching ketosis, but the number of AEDs did not change significantly before and after undertaking a KDT $[40,43]$. The age of patients has varied across studies, but even 6 to 10 -week-old neonates with RE are reported to tolerate KDTs well [42,50]. Intravenous (IV) KDT is recommended for patients with underlying concomitant ileus $[41,44,48]$, and early administration of IV KDT should be considered before switching to an enteral route [44]. There are no significant differences in the time to reach ketosis between IV and enteral routes, although hypertriglyceridemia and pancreatitis are frequently associated with IV KDT. Considering that critically ill patients with FIRES/SRSE receive many concurrent medications and are prone to malabsorption, IV KDT could be temporarily substituted for an enteral route [41]. Nonetheless, before evaluating the efficiency and safety of KDTs among FIRES/SRSE patients, factors such as concomitant treatments, variations in timing before the initiation of KDTs, the specific outcomes assessed, and possible publication bias should be considered [38].

\section{Use of KDTs for Infants with RE}

Contrary to a conventional view, under 2 years of age may be the optimum time to initiate a KDT because of the metabolic advantages of infants [50-55]. Numerous studies provide evidence for the advantages of using KDTs in infants with RE. Infants under 1.5 years of age have a higher chance of achieving seizure freedom than children over 1.5 years of age, and, interestingly, infants under 9 months of age also have a higher likelihood of achieving seizure freedom, demonstrating the ease of $\mathrm{KDT}$ administration and good outcomes before solid feeding [51]. These outcomes remained stable at long-term follow-up. Another study reports specific etiologic differences in long-term seizure-free outcomes among 115 patients who initiated KDTs before 1 year of age and shows that seizure freedom within the first 3 months could be a predictor of long-term seizure freedom [55]. A similar study including 109 patients with RE under 3 years of age with different etiologies reports that patients with a genetic etiology were particularly good responders to KDTs [54], with nearly half of patients with a confirmed genetic abnormality showing more than a 50\% reduction in seizure frequency. Nevertheless, most studies report a high efficacy of KDTs for some specific epilepsy syndromes such as WS [53,56-59], epilepsy with myoclonic atonic seizures [14], and Dravet syndrome [60]. Around two-thirds of patients with WS experience a reduction in seizures with $\mathrm{KDTs}$, and many show improvements in development, EEG activity, and number of concurrent AEDs [58]. Adrenocorticotropic hormone (ACTH) treatment is associated with a high responder rate and quick cessation of spasms, but it has higher rates of relapse and adverse effects than KDTs [53]. In a recent study comparing efficacy and safety between KDTs and standard high-dose ACTH treatment in WS infants [59], ACTH was associated with a higher rate of short-term remission among infants without prior treatment history of vigabatrin (VGB); however, it was also associated with a higher rate of relapse and similar rate of seizure-free outcome as KDTs at long-term follow-up. Also, KDTs had a higher rate of seizure freedom in longterm follow-up and a lower relapse rate in the short-term in infants with a prior treatment history of VGB. This study suggests that after VGB failure, a KDT could be a second-line treatment for WS.

\section{The Gut-Brain Axis and KDTs}

Several studies show that diversity in the diet significantly influences the composition of gut microbiota and the subsequent health of individuals [61]. Differences in the composition of gut microbiota between drug-sensitive/healthy control individuals and drug-resistant epilepsy patients indicates the possible involvement of dysbiosis in the development of drug-resistant epilepsy [62]. Dysbiosis may enhance susceptibility to seizures and accelerate illness resulting from chronic stress; thus, restoration and remodeling of a healthy gut microbial population could control seizures and boost quality of life $[63,64]$. In this regard, KDTs may positively impact seizures via alteration of the gut microbiota. In two mouse models, KDTs altered the composition of gut microbiota, including reducing bacterial alpha diversity and increasing certain bacteria [65] Moreover, high-dose antibiotics, which deplete gut microbiota, increase seizure vulnerability in wild-type and $\mathrm{Knal}^{-/-}$mice receiving KDTs. In this study, gut microbiota and KDTs with antiseizure 
properties were correlated with decreases in systemic gamma-glutamylated amino acid and enhanced $\gamma$-aminobutylic acid (GABA) levels in the hippocampus.

Similar to the results of animal studies, children with RE show alterations in the specific richness and diversity of gut microbiota, such as increased Bacteroidetes and decreased Firmicutes and Actinobacteria, after 6 months of KDT [66]. Moreover, the abundance of Clostridiales, Clostridia, Ruminococcaceae, Lachnospiraceae, Alistipes, and Rikenellaceae were significantly increased in those who failed to respond to KDT compared with good responders. The authors of this study speculated that specific microbiota might be therapeutic targets in the treatment of epilepsy and could serve as biomarkers indicating the efficacy of KDT.

\section{Triheptanoin}

Triheptanoin is a triglyceride composed of three heptanoate (C7 fatty acid) that is an artificial tasteless oil easily dissolved in food. Triheptanoin is used to treat many metabolic disorders because it has an anaplerotic role that replenishes substrates involved in the tricarboxylic acid (TCA) cycle and the ability to bypass metabolic blockade induced by enzyme deficiency [67]. Several studies using acute and chronic seizure mouse models demonstrate that triheptanoin exerts anti-seizure effects by increasing TCA intermediates and activating mitochondrial function, known as anaplerosis $[68,69]$. Calvert et al. [70] performed a study including 12 children with RE aged 3 to 18 years old and found that (1) children tolerated 30 to $100 \mathrm{~mL}$ triheptanoin per day (median, $55.5 \mathrm{~mL}$ ); (2) the most frequent adverse effect was gastrointestinal disturbance; (3) eight children completed the trial, of whom four safely completed an extended treatment period up to 909 days; (4) five children showed $>50 \%$ reduction in seizure frequency, including one patient who was seizure-free for 6 months; (5) children who previously received KDT showed better tolerance and outcomes than those who initiated KDT for the first time, presumably as a result of good parental compliance; and (6) no drug interactions were observed. Therefore, triheptanoin could possibly administered concurrently with AEDs. Another study in adults with RE reported that MCT or triheptanoin treatment was safe, feasible, and well tolerated as an add-on treatment [71]. In this double-blind study including 34 patients who took triheptanoin $(\mathrm{n}=17)$ or MCT oil $(\mathrm{n}=17)$ mixed into food, 11 and nine patients completed the study and showed good tolerance of the treatment at a median dose of 55 and $59 \mathrm{~mL}$ for 3 months, respectively, with reported side effects of diarrhea and abdominal pain. Although the aim of this study was not to investigate the efficacy of KDTs, it showed that MCT had good outcomes for focal un- aware seizures. Recent studies examining the use triheptanoin for treating Glut1DS [72-74] show that besides reducing glucose, its main benefit is a reduction in non-paroxysmal events.

\section{KDTs for Other Neurologic Disorders}

KDTs have been used for many neurologic disorders other than pediatric epilepsy, such as adult epilepsy, autism, Alzheimer's disease, and brain tumors [75]. The use of KDTs for treating autism is increasingly reported and, according to a systematic review in 2015, eight studies (five human and three animal) had tested the effect of KDTs in autism [76]. A prospective pilot study showed that 18 out of 30 children with autism showed better scores on an autism rating scale after KDT [77]. In addition, MCT was described as a potential supplement for mild Alzheimer's disease [78], and KDTs were proposed as a treatment for Down syndrome [79].

Another current interest is the potential anti-tumor effect of KDTs. Studies suggest that KDTs inhibit tumor cell growth by altering cell metabolism, which seems to enhance the response of other anti-tumor treatments $[80,81]$. A study with two glioma patients reported that KDTs as a monotherapy seemed ineffective in retarding tumor growth but had more promising effects when combined with standard treatments [82]. These patients received an energy-restricted $\mathrm{KD}$ that seemed effective in controlling the progression of primary brain tumors.

\section{Conclusion}

KDTs have diversified to alternative forms for different indications that can be selected based on the specific family and child situation. Recommendations for the use of KDTs have been strengthened by an increasing number of studies testing targeted therapeutic ranges.

\section{Conflicts of interest}

No potential conflict of interest relevant to this article was reported.

\section{ORCID}

Battamir Enkhtuy, https://orcid.org/0000-0001-6616-9552

Hye Eun Kwon, https://orcid.org/0000-0001-5180-4218

Heung Dong Kim, https://orcid.org/0000-0002-8031-7336 


\section{Author contributions}

Conceptualization: HDK. Data curation: BE and HEK. Writing-original draft: BE and HEK. Writing-review \& editing: HDK.

\section{References}

1. KossoffEH, Zupec-Kania BA, Auvin S, Ballaban-Gil KR, Christina Bergqvist AG, Blackford R, et al. Optimal clinical management of children receiving dietary therapies for epilepsy: updated recommendations of the International Ketogenic Diet Study Group. Epilepsia Open 2018;3:175-92.

2. DeVivo DC, Leckie MP, Ferrendelli JS, McDougal DB Jr. Chronic ketosis and cerebral metabolism. Ann Neurol 1978; 3:331-7.

3. Lutas A, Yellen G. The ketogenic diet: metabolic influences on brain excitability and epilepsy. Trends Neurosci 2013;36:32-40.

4. Youngson NA, Morris MJ, Ballard JWO. The mechanisms mediating the antiepileptic effects of the ketogenic diet, and potential opportunities for improvement with metabolism-altering drugs. Seizure 2017;52:15-19.

5. Ciarlone SL, Grieco JC, D’Agostino DP, Weeber EJ. Ketone ester supplementation attenuates seizure activity, and improves behavior and hippocampal synaptic plasticity in an Angelman syndrome mouse model. Neurobiol Dis 2016;96:38-46.

6. Sada N, Lee S, Katsu T, Otsuki T, Inoue T. Epilepsy treatment. Targeting LDH enzymes with a stiripentol analog to treat epilepsy. Science 2015;347:1362-7.

7. Viggiano A, Pilla R, Arnold P, Monda M, D’Agostino D, Coppola G. Anticonvulsant properties of an oral ketone ester in a pentylenetetrazole-model of seizure. Brain Res 2015;1618:50-4.

8. Kang HC, Lee HS, You SJ, Kang du C, Ko TS, Kim HD. Use of a modified Atkins diet in intractable childhood epilepsy. Epilepsia 2007; 48:182-6.

9. Kossoff EH, Cervenka MC, Henry BJ, Haney CA, Turner Z. A decade of the modified Atkins diet (2003-2013): results, insights, and future directions. Epilepsy Behav 2013;29:437-42.

10. Kossoff EH, McGrogan JR, Bluml RM, Pillas DJ, Rubenstein JE, Vining EP. A modified Atkins diet is effective for the treatment of intractable pediatric epilepsy. Epilepsia 2006;47:421-4.

11. Sharma S, Sankhyan N, Gulati S, Agarwala A. Use of the modified Atkins diet for treatment of refractory childhood epilepsy: a randomized controlled trial. Epilepsia 2013;54:481-6.

12. Kverneland M, Molteberg E, Iversen PO, Veierod MB, Tauboll E, Selmer KK, et al. Effect of modified Atkins diet in adults with drug-resistant focal epilepsy: a randomized clinical trial. Epilepsia 2018;59:1567-76.
13. Kossoff EH, Bosarge JL, Miranda MJ, Wiemer-Kruel A, Kang HC, Kim HD. Will seizure control improve by switching from the modified Atkins diet to the traditional ketogenic diet? Epilepsia 2010;51:2496-9.

14. Kim JA, Yoon JR, Lee EJ, Lee JS, Kim JT, Kim HD, et al. Efficacy of the classic ketogenic and the modified Atkins diets in refractory childhood epilepsy. Epilepsia 2016;57:51-8.

15. Amalou S, Gras D, Ilea A, Greneche MO, Francois L, Bellavoine $\mathrm{V}$, et al. Use of modified Atkins diet in glucose transporter type 1 deficiency syndrome. Dev Med Child Neurol 2016;58:1193-9.

16. Pfeifer HH, Thiele EA. Low-glycemic-index treatment: a liberalized ketogenic diet for treatment of intractable epilepsy. Neurology 2005;65:1810-2.

17. Kim SH, Kang HC, Lee EJ, Lee JS, Kim HD. Low glycemic index treatment in patients with drug-resistant epilepsy. Brain Dev 2017;39:687-92.

18. Muzykewicz DA, Lyczkowski DA, Memon N, Conant KD, Pfeifer HH, Thiele EA. Efficacy, safety, and tolerability of the low glycemic index treatment in pediatric epilepsy. Epilepsia 2009;50:1118-26.

19. Grocott OR, Herrington KS, Pfeifer HH, Thiele EA, Thibert RL. Low glycemic index treatment for seizure control in Angelman syndrome: a case series from the Center for Dietary Therapy of Epilepsy at the Massachusetts General Hospital. Epilepsy Behav 2017;68:45-50.

20. Thibert RL, Pfeifer HH, Larson AM, Raby AR, Reynolds AA, Morgan AK, et al. Low glycemic index treatment for seizures in Angelman syndrome. Epilepsia 2012;53:1498-502.

21. Liu YM. Medium-chain triglyceride (MCT) ketogenic therapy. Epilepsia 2008;49:33-6.

22. Liu YM, Wang HS. Medium-chain triglyceride ketogenic diet, an effective treatment for drug-resistant epilepsy and a comparison with other ketogenic diets. Biomed J 2013;36:9-15.

23. Neal EG, Chaffe H, Schwartz RH, Lawson MS, Edwards N, Fitzsimmons G, et al. A randomized trial of classical and medium-chain triglyceride ketogenic diets in the treatment of childhood epilepsy. Epilepsia 2009;50:1109-17.

24. Likhodii SS, Musa K, Mendonca A, Dell C, Burnham WM, Cunnane SC. Dietary fat, ketosis, and seizure resistance in rats on the ketogenic diet. Epilepsia 2000;41:1400-10.

25. Thavendiranathan P, Mendonca A, Dell C, Likhodii SS, Musa K, Iracleous $\mathrm{C}$, et al. The MCT ketogenic diet: effects on animal seizure models. Exp Neurol 2000; 161:696-703.

26. Chang P, Augustin K, Boddum K, Williams S, Sun M, Terschak JA, et al. Seizure control by decanoic acid through direct AMPA receptor inhibition. Brain 2016;139:431-43.

27. Chang P, Terbach N, Plant N, Chen PE, Walker MC, Williams 
RS. Seizure control by ketogenic diet-associated medium chain fatty acids. Neuropharmacology 2013;69:105-14.

28. Chang P, Zuckermann AM, Williams S, Close AJ, Cano-Jaimez M, McEvoy JP, et al. Seizure control by derivatives of medium chain fatty acids associated with the ketogenic diet show novel branching-point structure for enhanced potency. J Pharmacol Exp Ther 2015;352:43-52.

29. Hughes SD, Kanabus M, Anderson G, Hargreaves IP, Rutherford T, O'Donnell M, et al. The ketogenic diet component decanoic acid increases mitochondrial citrate synthase and complex I activity in neuronal cells. J Neurochem 2014;129:426-33.

30. Wlaz P, Socala K, Nieoczym D, Zarnowski T, Zarnowska I, Czuczwar SJ, et al. Acute anticonvulsant effects of capric acid in seizure tests in mice. Prog Neuropsychopharmacol Biol Psychiatry 2015;57:110-6.

31. Simeone TA, Matthews SA, Samson KK, Simeone KA. Regulation of brain PPARgamma2 contributes to ketogenic diet anti-seizure efficacy. Exp Neurol 2017;287:54-64.

32. Tan KN, Carrasco-Pozo C, McDonald TS, Puchowicz M, Borges $\mathrm{K}$. Tridecanoin is anticonvulsant, antioxidant, and improves mitochondrial function. J Cereb Blood Flow Metab 2017; 37:2035-48.

33. Rogawski MA. A fatty acid in the MCT ketogenic diet for epilepsy treatment blocks AMPA receptors. Brain 2016;139:3069.

34. Augustin K, Williams S, Cunningham M, Devlin AM, Friedrich M, Jayasekera A, et al. Perampanel and decanoic acid show synergistic action against AMPA receptors and seizures. Epilepsia 2018;59:e172-8.

35. Kossoff EH, Nabbout R. Use of dietary therapy for status epilepticus. J Child Neurol 2013;28:1049-51.

36. Nabbout R, Mazzuca M, Hubert P, Peudennier S, Allaire C, Flurin $\mathrm{V}$, et al. Efficacy of ketogenic diet in severe refractory status epilepticus initiating fever induced refractory epileptic encephalopathy in school age children (FIRES). Epilepsia 2010;51:2033-7.

37. Appavu B, Vanatta L, Condie J, Kerrigan JF, Jarrar R. Ketogenic diet treatment for pediatric super-refractory status epilepticus. Seizure 2016;41:62-5.

38. Arya R, Peariso K, Gainza-Lein M, Harvey J, Bergin A, Brenton JN, et al. Efficacy and safety of ketogenic diet for treatment of pediatric convulsive refractory status epilepticus. Epilepsy Res 2018;144:1-6.

39. Caraballo RH, Flesler S, Armeno M, Fortini S, Agustinho A, Mestre G, et al. Ketogenic diet in pediatric patients with refractory focal status epilepticus. Epilepsy Res 2014;108:1912-6.

40. Park EG, Lee J, Lee J. The ketogenic diet for super-refractory sta- tus epilepticus patients in intensive care units. Brain Dev 2019;41:420-7.

41. Chiusolo F, Diamanti A, Bianchi R, Fusco L, Elia M, Capriati T, et al. From intravenous to enteral ketogenic diet in PICU: a potential treatment strategy for refractory status epilepticus. Eur J Paediatr Neurol 2016;20:843-7.

42. Cobo NH, Sankar R, Murata KK, Sewak SL, Kezele MA, Matsumoto JH. The ketogenic diet as broad-spectrum treatment for super-refractory pediatric status epilepticus: challenges in implementation in the pediatric and neonatal intensive care units. J Child Neurol 2015;30:259-66.

43. Farias-Moeller R, Bartolini L, Pasupuleti A, Brittany Cines RD, Kao A, Carpenter JL. A practical approach to ketogenic diet in the pediatric intensive care unit for super-refractory status epilepticus. Neurocrit Care 2017;26:267-72.

44. Lin JJ, Lin KL, Chan OW, Hsia SH, Wang HS; CHEESE Study Group. Intravenous ketogenic diet therapy for treatment of the acute stage of super-refractory status epilepticus in a pediatric patient. Pediatr Neurol 2015;52:442-5.

45. O'Connor SE, Ream MA, Richardson C, Mikati MA, Trescher $\mathrm{WH}$, Byler DL, et al. The ketogenic diet for the treatment of pediatric status epilepticus. Pediatr Neurol 2014;50:101-3.

46. Peng P, Peng J, Yin F, Deng X, Chen C, He F, et al. Ketogenic diet as a treatment for super-refractory status epilepticus in febrile infection-related epilepsy syndrome. Front Neurol 2019; 10:423.

47. Sort R, Born AP, Pedersen KN, Fonsmark L, Uldall P. Ketogenic diet in 3 cases of childhood refractory status epilepticus. Eur J Paediatr Neurol 2013;17:531-6.

48. Strzelczyk A, Reif PS, Bauer S, Belke M, Oertel WH, Knake S, et al. Intravenous initiation and maintenance of ketogenic diet: proof of concept in super-refractory status epilepticus. Seizure 2013;22:581-3.

49. Nam SH, Lee BL, Lee CG, Yu HJ, Joo EY, Lee J, et al. The role of ketogenic diet in the treatment of refractory status epilepticus. Epilepsia 2011;52:e181-4.

50. Thompson L, Fecske E, Salim M, Hall A. Use of the ketogenic diet in the neonatal intensive care unit-Safety and tolerability. Epilepsia 2017;58:e36-9.

51. Dressler A, Trimmel-Schwahofer P, Reithofer E, Groppel G, Muhlebner A, Samueli S, et al. The ketogenic diet in infants: advantages of early use. Epilepsy Res 2015;116:53-8.

52. Ismayilova N, Leung MA, Kumar R, Smith M, Williams RE. Ketogenic diet therapy in infants less than two years of age for medically refractory epilepsy. Seizure 2018;57:5-7.

53. Kossoff EH, Hedderick EF, Turner Z, Freeman JM. A case-control evaluation of the ketogenic diet versus ACTH for new-on- 
set infantile spasms. Epilepsia 2008;49:1504-9.

54. Kim SH, Shaw A, Blackford R, Lowman W, Laux LC, Millichap JJ, et al. The ketogenic diet in children 3 years of age or younger: a 10-year single-center experience. Sci Rep 2019;9:8736.

55. Riantarini I, Kim HD, Ko A, Kim SH, Kang HC, Lee JS, et al. Short- and long-term seizure-free outcomes of dietary treatment in infants according to etiology. Seizure 2019;71:100-4.

56. Eun SH, Kang HC, Kim DW, Kim HD. Ketogenic diet for treatment of infantile spasms. Brain Dev 2006;28:566-71.

57. Pires ME, Ilea A, Bourel E, Bellavoine V, Merdariu D, Berquin P, et al. Ketogenic diet for infantile spasms refractory to first-line treatments: an open prospective study. Epilepsy Res 2013; 105:189-94.

58. Hong AM, Turner Z, Hamdy RF, Kossoff EH. Infantile spasms treated with the ketogenic diet: prospective single-center experience in 104 consecutive infants. Epilepsia 2010;51:1403-7.

59. Dressler A, Benninger F, Trimmel-Schwahofer P, Groppel G, Porsche B, Abraham K, et al. Efficacy and tolerability of the ketogenic diet versus high-dose adrenocorticotropic hormone for infantile spasms: a single-center parallel-cohort randomized controlled trial. Epilepsia 2019;60:441-51.

60. Dressler A, Trimmel-Schwahofer P, Reithofer E, Muhlebner A, Groppel G, Reiter-Fink E, et al. Efficacy and tolerability of the ketogenic diet in Dravet syndrome: comparison with various standard antiepileptic drug regimen. Epilepsy Res 2015; 109:81-9.

61. David LA, Maurice CF, Carmody RN, Gootenberg DB, Button JE, Wolfe BE, et al. Diet rapidly and reproducibly alters the human gut microbiome. Nature 2014;505:559-63.

62. Peng A, Qiu X, Lai W, Li W, Zhang L, Zhu X, et al. Altered composition of the gut microbiome in patients with drug-resistant epilepsy. Epilepsy Res 2018;147:102-7.

63. He Z, Cui BT, Zhang T, Li P, Long CY, Ji GZ, et al. Fecal microbiota transplantation cured epilepsy in a case with Crohn's disease: the first report. World J Gastroenterol 2017;23:3565-8.

64. Braakman HMH, van Ingen J. Can epilepsy be treated by antibiotics? J Neurol 2018;265:1934-6.

65. Olson CA, Vuong HE, Yano JM, Liang QY, Nusbaum DJ, Hsiao EY. The gut microbiota mediates the anti-seizure effects of the ketogenic diet. Cell 2018;173:1728-41.

66. Zhang Y, Zhou S, Zhou Y, Yu L, Zhang L, Wang Y. Altered gut microbiome composition in children with refractory epilepsy after ketogenic diet. Epilepsy Res 2018;145:163-8.

67. Borges K, Sonnewald U. Triheptanoin: a medium chain triglyceride with odd chain fatty acids: a new anaplerotic anticonvulsant treatment? Epilepsy Res 2012;100:239-44.

68. Kim TH, Borges K, Petrou S, Reid CA. Triheptanoin reduces seizure susceptibility in a syndrome-specific mouse model of generalized epilepsy. Epilepsy Res 2013;103:101-5.

69. Willis S, Stoll J, Sweetman L, Borges K. Anticonvulsant effects of a triheptanoin diet in two mouse chronic seizure models. Neurobiol Dis 2010;40:565-72.

70. Calvert S, Barwick K, Par M, Ni Tan K, Borges K. A pilot study of add-on oral triheptanoin treatment for children with medically refractory epilepsy. Eur J Paediatr Neurol 2018;22:107480.

71. Borges K, Kaul N, Germaine J, Kwan P, O’Brien TJ. Randomized trial of add-on triheptanoin vs medium chain triglycerides in adults with refractory epilepsy. Epilepsia Open 2019;4:15363.

72. Hainque E, Gras D, Meneret A, Atencio M, Luton MP, Barbier $\mathrm{M}$, et al. Long-term follow-up in an open-label trial of triheptanoin in GLUT1 deficiency syndrome: a sustained dramatic effect. J Neurol Neurosurg Psychiatry 2019;90:1291-3.

73. Mochel F, Hainque E, Gras D, Adanyeguh IM, Caillet S, Heron $\mathrm{B}$, et al. Triheptanoin dramatically reduces paroxysmal motor disorder in patients with GLUT1 deficiency. J Neurol Neurosurg Psychiatry 2016;87:550-3.

74. Pascual JM, Liu P, Mao D, Kelly DI, Hernandez A, Sheng M, et al. Triheptanoin for glucose transporter type I deficiency (G1D): modulation of human ictogenesis, cerebral metabolic rate, and cognitive indices by a food supplement. JAMA Neurol 2014;71:1255-65.

75. Stafstrom CE, Rho JM. The ketogenic diet as a treatment paradigm for diverse neurological disorders. Front Pharmacol 2012;3:59.

76. Castro K, Faccioli LS, Baronio D, Gottfried C, Perry IS, dos Santos Riesgo R. Effect of a ketogenic diet on autism spectrum disorder: a systematic review. Res Autism Spectr Disord 2015; 20:31-8.

77. Evangeliou A, Vlachonikolis I, Mihailidou H, Spilioti M, Skarpalezou A, Makaronas N, et al. Application of a ketogenic diet in children with autistic behavior: pilot study. J Child Neurol 2003;18:113-8.

78. Herbert MR, Buckley JA. Autism and dietary therapy: case report and review of the literature. J Child Neurol 2013;28:97582.

79. Taylor MK, Sullivan DK, Mahnken JD, Burns JM, Swerdlow RH. Feasibility and efficacy data from a ketogenic diet intervention in Alzheimer's disease. Alzheimers Dement (N Y) 2017;4:28-36.

80. Kaneko KN, Wong M, Corley MJ, Lee RWY. The ketogenic diet as a potential therapy in Down syndrome. J Pediatr Pediatr Med 2018;2:11-7. 
81. Klement RJ, Champ CE, Otto C, Kammerer U. Anti-tumor effects of ketogenic diets in mice: a meta-analysis. PLoS One 2016;11:e0155050.

82. Schwartz K, Chang HT, Nikolai M, Pernicone J, Rhee S, Olson
$\mathrm{K}$, et al. Treatment of glioma patients with ketogenic diets: report of two cases treated with an IRB-approved energy-restricted ketogenic diet protocol and review of the literature. Cancer Metab 2015;3:3. 